\title{
Oncocytic Carcinoma of the Nasal Septum, A Rare Cause of Unilateral Epistaxis
}

\author{
Hypte Raymund V. Aujero, ${ }^{1}$ Kathleen Makrina R. Fellizar-Lopez, ${ }^{1}$ Arsenio Claro A. Cabungcal, ${ }^{1}$ \\ Mark Angelo C. Ang² and Dahlia Teresa R. Argamosa² \\ ${ }^{1}$ Department of Otorhinolaryngology, College of Medicine and Philippine General Hospital, University of the Philippines Manila \\ ${ }^{2}$ Department of Pathology, College of Medicine, University of the Philippines Manila
}

\begin{abstract}
A case of a 74-year-old male with unilateral nasal obstruction, recurrent epistaxis and a right intranasal mass is presented. It was initially diagnosed as hemangioma but final histopathology report revealed oncocytic carcinoma. Oncocytic carcinoma is a rare tumor of the salivary glands with very few reported cases, most of which involve the parotid gland. It has a tendency to recur with inadequate excision. Diagnosis is histopathologic. It is to be emphasized that adequate tissue samples should be taken in order to provide a definite diagnosis from biopsy, and subsequently institute proper definitive management.
\end{abstract}

Key Words: oncocytic carcinoma, nasal septum, case report

\section{Introduction}

Carcinomas of the sinonasal tract account for approximately $1 \%$ of human malignancies. There is a strong predilection for Caucasian males and most of them are more than 50 years old. Rarer still are carcinomas of the oncocytic type in this area, with only sixteen being reported earlier. ${ }^{1-5}$

Oncocytomas account for fewer than $1 \%$ of all salivary gland tumors. They are usually benign tumors that arise most commonly in the parotid gland but can also originate in the other salivary glands. The cells of origin, oncocytes, are large epithelial cells with granular eosinophilic cytoplasm caused by mitochondrial hyperplasia. Oncocytes can be found in otherwise normal major or minor salivary glands. They are also found in the pancreas, respiratory tract, thyroid, parathyroid, pituitary, adrenal glands, and kidney, although the majority of tumors occur in the superficial lobe of the parotid gland. The malignant variety occurs at less than five percent of all oncocytomas in the salivary gland. ${ }^{6}$ \footnotetext{
15-19, 2010, Lotte Hotel, Seoul, Korea.

Corresponding author: Hypte Raymund V. Aujero, MD Department of Otorhinolaryngology

Philippine General Hospital

University of the Philippines Manila

Taft Avenue, Ermita, Manila 1000 Philippines

Telephone: $+6325264360 / 5548400$ local 2152

Email: drhrva@yahoo.com
}

Presented at the 4th Congress of International Federation of Head and Neck Oncologic Societies: Shifting Paradigms in Head and Neck Oncology, June
We present a rare case of oncocytic carcinoma of the nasal cavity, wherein an earlier pre-operative biopsy was diagnosed as hemangioma.

\section{Case Report}

A 74-year-old male presented at the Otorhinolaryngology clinic at the Outpatient Department of the Philippine General Hospital with a complaint of unilateral epistaxis of one year and seven months duration. Prior to consulting in our institution, the patient was admitted four times at a local hospital for unilateral nasal bleeding amounting to approximately three cups in each episode. His epistaxis was attributed to hypertension and anterior nasal packing was done to control the bleeding.

Eighteen months after the first episode, he consulted in our institution's outpatient department where a violaceous mass was seen in the right nasal cavity. Yellowish discharge from the same side and septal deviation on the contralateral side were also noted. Pre-operative chest X-ray and liver function tests showed no signs of distant metastasis. No neck nodes were palpated on physical exam. Paranasal Computed Tomography (CT) showed a non-enhancing mass in the right nasal cavity, septal deviation, and pansinusitis. It was also noted that the mass was invading the ethmoid cells. No lymphadenopathies were seen on CT. Pre-operative biopsy of the mass was initially read as hemangioma. The mass was eventually excised via an endoscopic approach a month later. Intraoperatively, the mass was seen attached to and perforating the posterior septum at the right nasal cavity, abutting the middle turbinate of the left nasal cavity. Microscopic sections of the mass showed oncocytes invading vascular and lymphatic spaces. Thus, the final diagnosis was Oncocytic Carcinoma Stage II T2N0M0. Endoscopic septectomy and middle turbinectomy were also subsequently performed with the excised specimens turning out to be negative for tumor involvement.

\section{Discussion}

In a patient with significant recurrent epistaxis and a unilateral mass in the nasal cavity, several entities have to be considered. Benign tumors commonly considered are antrochoanal polyps, inverted papillomas, hemangiomas, 
pyogenic granulomas, and angiofibromas. ${ }^{6}$ But in a 74-yr-old male who is a former smoker, one would lean towards a diagnosis of carcinoma, with squamous cell carcinoma being the primary consideration. ${ }^{5}$ After a thorough history and physical examination, imaging and biopsy of the growth should be performed. A CT scan of the para-nasal sinuses was obtained to assess the extent of tumor growth and involvement of adjacent structures. Figure 1 shows the mass to be confined to the right nasal cavity, compressing the bony structures, but with no intracranial extension. The mass was noted to be non-enhancing on contrast, pointing to a non-vascular tumor. Considering the patient's age, findings on physical exam and CT scan, we may rule-out angiofibromas, antrochoanal polyps, and hemangiomas. A punch biopsy was done to determine the histopathologic diagnosis. The biopsy specimen showed prominent vascularity with numerous hemosiderin-laden macrophages and was thus read as hemangioma. Excision of the entire mass was therefore performed. Intra-operatively, the mass was well-encapsulated, dark violet in color, and was noted to be attached to the septum and perforating into the left nasal cavity.

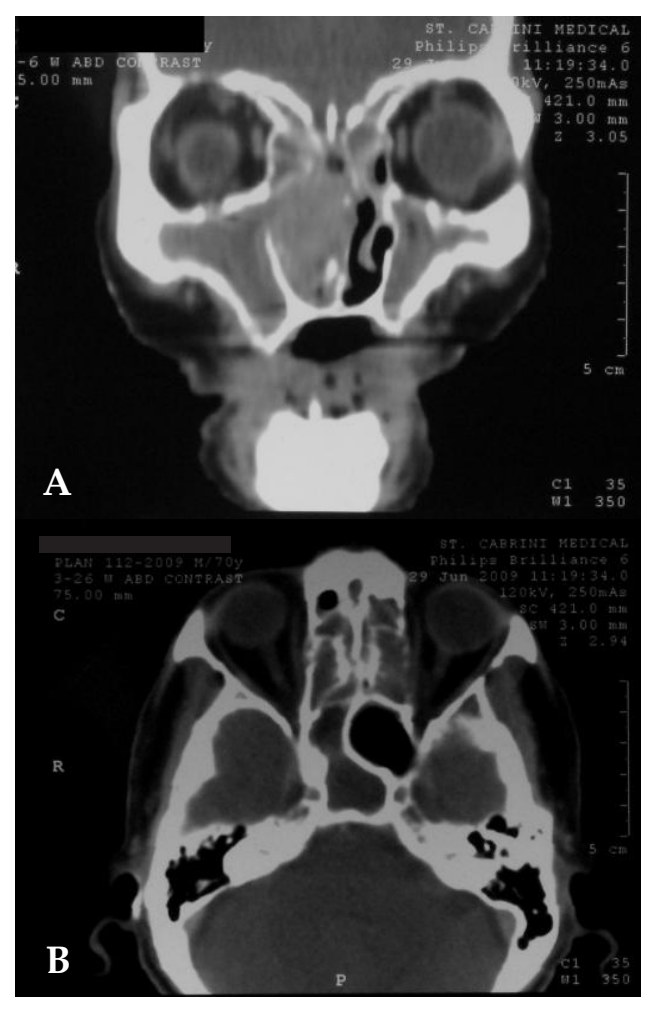

Figure 1. CT showing mass in the right nasal cavity (coronal view, A and axial view, B)

Microscopic sections of the definitive specimen revealed polygonal cells with round eccentrically located basophilic nuclei, with some nuclei having an open chromatin pattern, while others had dense chromatin patterns. Nucleoli were noted to be small. The cells have abundant granular eosinophilic cytoplasm indicative of an abundance of mitochondria. Such findings are characteristic of oncocytomas. ${ }^{7}$ Furthermore, angiolymphatic invasion, as seen in Figure 2, points to a diagnosis of a malignancy.

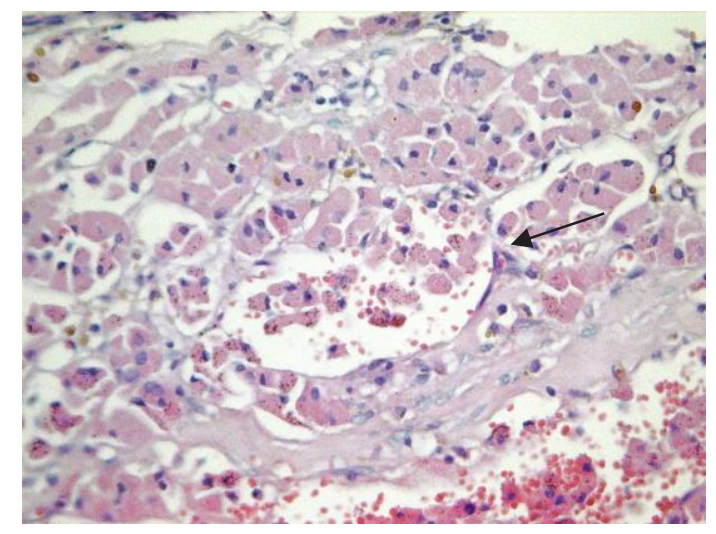

Figure 2. Arrow points to angiolymphatic invasion of the tumor cells.

Upon review of the slides, hemosiderin-laden macrophages were also noted to be abundant. These findings in a background of high vascularity was what led to the diagnosis of hemangioma in the pre-operative punch biopsy. The discrepancy in the diagnosis between the previous punch biopsy and the definitive specimen is thus attributed to a sampling error. The previous biopsy may have been taken from the superficial tissues surrounding the mass itself. The hemangioma-like appearance of the previous biopsy may have been part of a reactive response of the surrounding tissue to the actual mass.

Oncocytomas are benign tumors most commonly seen in salivary glands, most often in the parotid. The malignant variant is termed malignant oncocytoma or oncocytic carcinoma and is characterized by invasion of blood vessels and lymphatics by the tumor cells.

Oncocytic carcinomas represent less than $1 \%$ of salivary gland tumors. Men are affected in two-thirds of cases with a wide age range of 25-91 yrs and mean of 62.5 years. Nearly $80 \%$ of these tumors involve the parotid gland, $8 \%$ involve the submandibular gland, and the rest involve the minor salivary glands. These are high grade tumors characterized by multiple local recurrences and regional and/or distant metastases with latter being the most important prognostic indicator. ${ }^{8}$ Oncocytic carcinoma is rare and rarer still are those found in the sinonasal tract with only sixteen being reported previously in the literature. ${ }^{1-5}$

Malignant oncocytomas are managed by complete surgical excision. Radiotherapy is not usually a mainstay in treatment as oncocytes are radioresistant, 3,8 though it has been shown to reduce recurrence for parotid malignant oncocytomas. ${ }^{5}$ Nayak and colleagues had their patient 
treated with 60 Gy in 28 fractions. ${ }^{1}$ No presence of tumor was seen in 6 months. Lombardi and colleagues subjected their patient with $60 \mathrm{~Gy}$ and was reported to be cancer free for 3 years. $^{3}$ Of the 16 cases in the literature reviewed, 8 recurred. These patients had further surgical management and some underwent radiotherapy as adjunct treatment. Of the eight, 5 were tumor-free on follow-up. ${ }^{3,5}$ Neck dissection is mandatory only when nodal metastases and/or a nodal recurrence is present. Elective neck dissection should be reserved for tumors that are at high risk for occult nodal metastases due to their location, like in the upper jaw, or in advanced stages and/or in cases showing biologic aggressiveness. ${ }^{5}$ In this particular case, neck dissection was not done.

\section{Conclusion}

Oncocytic carcinoma is a rare tumor of the salivary glands with very few reported cases, most of which involve the parotid gland. It has a tendency to recur with inadequate excision. Diagnosis is histopathologic. It should be emphasized that adequate and representative tissue samples should be taken in order to provide an accurate diagnosis from biopsies, and to subsequently institute proper definitive management. Complete resection is the mainstay of management, with radiotherapy and neck dissection not routinely done. The possibility of recurrences mandates prolonged follow-up.

\section{References}

1. Nayak, DR, Pillai S, Balakrishnan R, Thomas R, Rao R. Malignant oncocytoma of the nasal cavity: a case report. Am J Otolaryngol. 1999; 20(5):323-7.

2. DiMaio SJ, DiMaio VJ, DiMaio TM, Nicastri AD, Chen CK. Oncocytic carcinoma of the nasal cavity. South Med J. 1980; 73(6):803-6.

3. Lombardi D, Piccioni M, Farina D, Morassi ML, Nicolai P. Oncocytic carcinoma of the maxillary sinus: a rare neoplasm. Eur Arch Otorhinolaryngol. 2006; 263(6):528-31.

4. Abe T, Murakami A, Nakajima N, et al. Oncocytic carcinoma of the nasal cavity with widespread lymph node metastases. Auris Nasus Larynx. 2007; 34(3):393-6.

5. Adhikari P, Pradhananga RB, Sinha BK, Pradhan B, Thapa N. Oncocytoma of maxillary sinus--a rare presentation. Nepal Med Coll J. 2006; 8(4):292-3.

6. Hanna EY, Lee S, Fan CY, Suen JY. Benign Neoplasms of the Salivary Glands. In: Cummings CW, Flint PW, Harker LA, Haughey BH, Richardson MA, Robbins KT, Thomas JR, Schuller DE, eds. Otolaryngology Head \& Neck Surgery, $4^{\text {th }}$ ed. Pennsylvania: Elsevier Mosby; 2005. pp. 1348-1377.

7. Hoffman H, Funk G, Endres D. Evaluation and Surgical Treatment of Tumors of the Salivary Glands. In: Thawley SE, Panje WR, Batsakis JG, Lindberg RD, eds. Comprehensive Management of Head and Neck Tumors. Philadelphia, Pennsylvania: WB Saunders Company; 1999. pp. 1147-1181.

8. Sciubba JJ, Shimono M. Oncocyitc Carcinoma. In: Barnes L, Eveson JW, Reichart P, Sidransky D, eds. World Health Organization Classification of Tumors: Pathology and Genetics of Head and Neck Tumors. Lyon, France: International Agency for Research on Cancer (IARC) Press; 2005. p. 235.

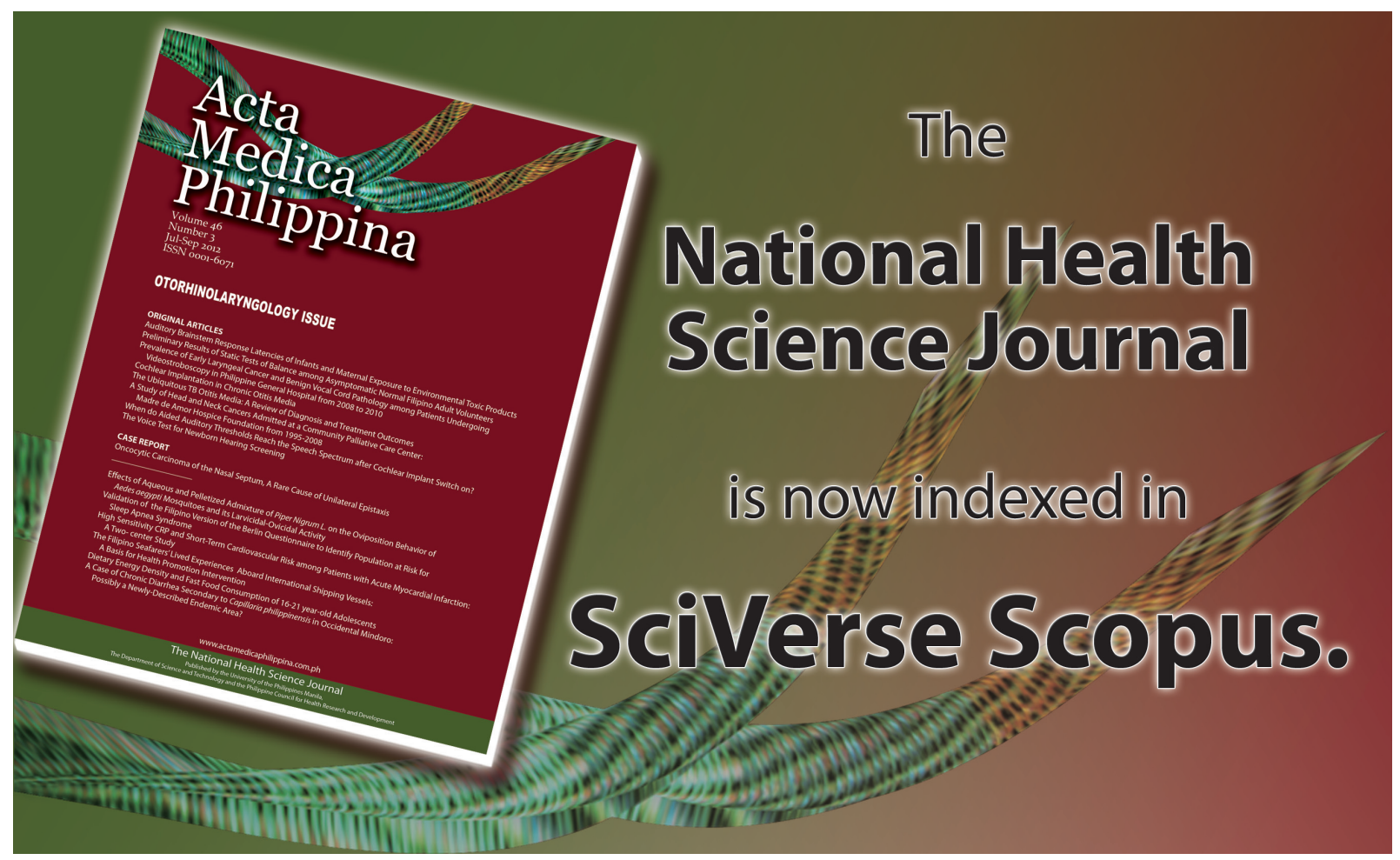

